\title{
Téoros
}

Revue de recherche en tourisme

\section{Rêves de Moyen-Orient et Moyen-Orient de rêve}

Le tourisme entre réalisme et exotisme

\section{Mimoun Hillali}

Volume 25, numéro 2, été 2006

Désirs d’Orient : du passé vers l'avenir

URI : https://id.erudit.org/iderudit/1071061ar

DOI : https://doi.org/10.7202/1071061ar

Aller au sommaire du numéro

Éditeur(s)

Université du Québec à Montréal

ISSN

0712-8657 (imprimé)

1923-2705 (numérique)

Découvrir la revue

Citer cet article

Hillali, M. (2006). Rêves de Moyen-Orient et Moyen-Orient de rêve : le tourisme entre réalisme et exotisme. Téoros, 25(2), 5-12.

https://doi.org/10.7202/1071061ar d'utilisation que vous pouvez consulter en ligne.

https://apropos.erudit.org/fr/usagers/politique-dutilisation/ 


\section{Rêves de Moyen-Orient et Moyen-Orient de rêve Le tourisme entre réalisme et exotisme}

\section{Mimoun Hillali}

Que serait le voyage d'agrément sans le foisonnement des rêves qui le précède? Et que serait le rêve lui-même sans la fertilité de l'imaginaire qui l'alimente avec une étonnante persévérance ? Dans le domaine du tourisme, l'imaginaire a un pouvoir surprenant sur le touriste. "Partir en vacances, c'est mettre en œuvre un projet de plaisir qui était cantonné dans le registre imaginaire » (Lauru et al., 2004 : 36). Ce dernier ravitaille en sensations, en perceptions, en impressions et en représentations tout candidat au voyage. II faut admettre qu'il existe des situations où le touriste potentiel se projette virtuellement dans sa "destination de rêve " et s'octroie par procuration des agréments qui font partie des préparatifs psychiques ou des rites initiateurs du voyage. N'a-t-on pas en tête, et presque en permanence, l'image affectionnée d'un Eldorado proche ou lointain qui sommeille au fond d'une espérance en dormance?

L'aspiration au voyage se manifeste virtuellement à travers une "projection représentation » qui chérit inlassablement une "destination promise " et souvent différée, faute de moyens ou de circonstances favorables. Alors l'espoir garde toutes ses promesses. Certains lieux, chargés d'histoire et de légendes, tel le Moyen-Orient, offrent à l'imagination des fertilisants agrémentés par l'attente d'un voyage. Celui-ci permet, paradoxalement, de faire de cette attente un ravissement entretenu par le souhait de partir un jour. C'est que l'espérance possède la bienheureuse faculté de minimiser les problèmes, en atténuant une somme insoupçonnée de tensions, de stress et de routines. "Qu'elle est étouffante, la vie, sans l'immensité de l'espoir ${ }^{1}$ ! » L'imaginaire se charge, en plus de l'euphorie thérapeutique qu'il procure à l'esprit, de préparer le visiteur à l'épreuve de ressourcement, le temps venu, pour y puiser toute l'énergie nécessaire à son épanouissement. Tout est destiné à la diminution des surcharges stressantes sécrétées par la contrainte du travail ou la frustration du quotidien. L'inconvénient de ces évasions «touristiquement » correctes et psychiquement profitables est que le déplacement, sa trajectoire et sa durée n'ont qu'un effet éphémère... mais quelle formidable satisfaction! Le départ à lui seul devient, en quelque sorte, une fin en soi! Dans pareilles circonstances, la qualité, la renommée ou l'attrait du lieu du séjour s'éclipse face à la force de la répulsion du milieu du labeur ou de la résidence et, surtout, devant la modestie des moyens à consacrer aux vacances. Seule la possibilité de partir compte et dérobe à la vigueur de la destination tout le charme du fantasme ${ }^{2}$ qui célèbre le mariage d'amour entre la méditation du touriste " engagé » et la destination qui hante ses rêves.

C'est dans cette approche énigmatique du rêve et de l'imaginaire touristiques que le Proche et le Moyen-Orient exercent une émotion fascinante sur les touristes passionnés par l'historicité et la culturalité des lieux géographiques ou par la piété et la spiritualité des milieux socioculturels; d'autres visiteurs sont séduits par la béatitude d'un contexte sociogéographique hautement antique, ce qui explique que les touristes, malgré des effervescences responsables d'insécurité, s'y rendent dans un élan qui rappelle, par certains aspects du voyage, un engouement qui oscille entre réalisme et exotisme.

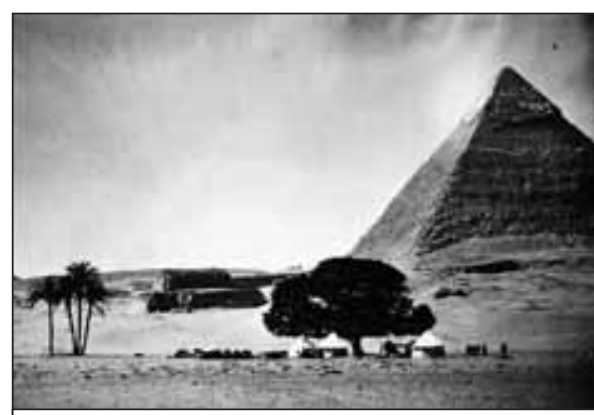

Pyramide de Gizeh et çampement des voyageurs vers 1868 (Égypte).

Photo : Albert Goupil/ @ Bibliothèque nationale de France

\section{Des lieux et des rêves pour des moments de trêve : réflexions préliminaires au voyage d'Orient}

Assurément, le tourisme d'agrément, lorsqu'il est fondé sur le raisonnement, a quelque chose de culturel, de spirituel et même de sensuel au sens voluptueux du terme. Dès lors, en dehors du voyage d'ordre « anti-cafard » qui ne répond que partiellement aux invocations que suscite le tourisme d'agrément $^{3}$ médité longtemps à l'avance, y aurait-il des voyages touristiques sans fiction ou sans fantasmes, sans imagination ou sans illusions? À première vue, non; surtout lorsqu'il s'agit d'une première visite, quelle que soit la destination.

Partir par amour visiter un pays, une localité ou un lieu comporte quelques secrets impénétrables! Ce n'est pas un voyage comme les autres, car il ne découle pas de choix hâtifs ou de désirs naïfs, encore moins d'une mode ou d'une quelconque contrainte; ce n'est pas non plus un déplacement ordinaire au sens strict de la mobilité physique à des fins ludiques, esthétiques ou corporelles (bronzage, cure, sport, jeu...). Encore faut-il être en mesure de pouvoir palper l'essence de ces mouvements à l'intérêt évasif. D'emblée, le jeu est faussé lorsque l'obser- 
vateur se contente de l'interprétation des images envahissantes proposées par une panoplie de clichés véhiculés par des concepts forgés par les spécialistes de la publicité et qui contribuent à la « taylorisation » des imaginaires. "Aussi, nous offre-t-on une nature aseptisée et toute une infrastructure qui élimine l'imprévisible et qui gère jusqu'à la liberté [...] Même si l'on voit les choses sous cet angle, faut-il pour autant jouer les rabat-joie? » (Louis, 1990 : 11). À ce propos, il est une catégorie de voyages d'inspiration particulière, où la quête d'un hédonisme parfait, au sens platonique de l'expression, propulse le voyageur dans une sorte de transe à la limite de l'aliénation réconfortante. Cette recherche persistante d'un idéal consolateur, dans son acception idyllique sur le plan sociologique, philosophique ou théologique, donne à ce type de tourisme toute sa dimension féerique enjouée, à la fois rituelle, cultuelle et culturelle.

La recherche d'une forme de genèse immémoriale fait que tout retour aux sources se veut une prospection de son «soi » métaphysique par son «soi » pieux ou philanthrope, didactique ou assoiffé de savoir. Le fait de se projeter dans un espace originel, ou censé l'être malgré les aléas du temps, a pour résultat immédiat de sceller la réconciliation entre la cogitation de l'esprit (foyer sensoriel de la réflexion) et la quiétude de l'âme (émanation vitale de l'ardeur). Toutefois, "l'esprit peut être partie prenante d'un lieu, et rester tout à fait libre dans l'espace" (Maffesoli, 2003: 44). Partir à la quête des fondements séculaires de la civilisation humaine, impalpables dans leur essence éthique ou mythique, matériellement parlant, mais tangibles par l'intensité de leur contexte historique, de leur production patrimoniale et de leur configuration géographique, fait partie des sentiments immanents de la nature humaine et des aspirations intrinsèques à la culture du voyage.

Que dire, ou comment décrire, des sentiments intangibles mais vécus, que suscitent la mise en scène de la découverte d'une destination glorifiée et la ferveur du voyageur contrit, qui voit enfin se réaliser son rêve en foulant pour la première fois le sol du lieu mythifié ? Comment retracer ce moment tant attendu qui symbolise l'accomplissement d'un rêve? Car la perception préalable et la vision réelle d'une destination sacralisée, parfois divinisée, procurent des sensations qu'aucun écrit n'est en mesure de restituer de manière concordante. Peut-on pénétrer

Tableau 1

Émotion, perception et concentration au moment de la visite d'un haut lieu historique ou théologique selon les types de touristes

\begin{tabular}{l|l|l|l}
\hline Sensation & Émotion & Perception & Concentration \\
\hline Touriste pieux & $\begin{array}{l}\text { Forte émotion exprimée } \\
\text { Souvent par des prières }\end{array}$ & $\begin{array}{l}\text { Sacrée, rituelle, cultuelle et } \\
\text { spirituelle }\end{array}$ & $\begin{array}{l}\text { Forte et durable durant } \\
\text { tout le séjour }\end{array}$ \\
Touriste mixte & $\begin{array}{l}\text { Émotion variable avec une } \\
\text { bonne curiosité profane }\end{array}$ & $\begin{array}{l}\text { Spirituelle, culturelle et source } \\
\text { de méditation }\end{array}$ & $\begin{array}{l}\text { Forte ou discontinue par } \\
\text { endroits et par moments }\end{array}$ \\
\hline Touriste profane & $\begin{array}{l}\text { Éblouissement et recherche de } \\
\text { repères cognitifs }\end{array}$ & $\begin{array}{l}\text { Culturelle, didactique et } \\
\text { authentique }\end{array}$ & $\begin{array}{l}\text { Forte au début de la } \\
\text { visite, elle décline à la fin }\end{array}$ \\
\hline
\end{tabular}

Source : Données de l'auteur.

\section{Tableau 2}

Pèlerinage ou tourisme le sentiment et le raisonnement

\begin{tabular}{|c|c|c|c|c|}
\hline \multicolumn{2}{|c|}{ Pèlerinage } & \multirow{2}{*}{$\begin{array}{c}\text { Mixte } \\
\text { Pèlerin = touriste }\end{array}$} & \multicolumn{2}{|c|}{ Tourisme } \\
\hline Touriste pieux & Pèlerin > touriste & & Touriste < pèlerin & Touriste laïc \\
\hline \multicolumn{3}{|c|}{ Sacré $<1$} & + & - $>$ Profan \\
\hline
\end{tabular}

dans l'essence de l'imaginaire d'un prieur juif qui se prosterne devant le Mur des lamentations, d'un chrétien qui prie intensément dans l'église de la Nativité ou d'un musulman qui effectue avec ferveur son pèlerinage à la Mecque? Dans ces cas, pour ceux qui viennent de loin, la majorité des croyants réfutent le terme même de touriste et lui préfèrent celui de pèlerin!

Faut-il rappeler, surtout en ces temps de conflits insensés aux yeux des pacifistes, des non-violents et des philanthropes, que le Moyen-Orient représente un lieu unique au monde ; un lieu chargé, jusqu'au seuil de la saturation, de symboles, de mémoires et de mythes confits dans un immense héritage fait de créations humaines et de "révélations divines "? C'est donc une destination bénie des dieux, si toutefois le monothéisme universel autorise le recours à cette expression polythéiste. L'histoire et la légende y ont produit, dans une concurrence loyale, les premières œuvres scientifiques, artistiques, littéraires, juridiques et mythiques aussi. Les mythes et les réalités de ces triomphes millénaires, dont l'écho et l'utilité ont été répandus par la conquête, le commerce ou la religion aux quatre coins du monde, meublent encore notre imaginaire.

La densité des patrimoines, la pluralité des cultures et la diversité des confessions y sont un fait saillant incontournable. L'imaginaire qui motive les voyages du Moyen-Orient s'alimente à des sources polyvalentes. Les décideurs le savent bien. Mais il arrive que la consistance des profits à tirer des richesses régionales, quitte à recourir à la force, avec son lot d'abus ou de violences, fasse oublier le respect élémentaire dû au statut historique de cette région. Les acteurs du tourisme, promoteurs et voyageurs, doivent en tenir compte à l'avenir, quelle que soit l'action à entreprendre, y compris les opérations à but non lucratif !

La force de l'imaginaire! On s'imagine que l'imaginaire, c'est léger... C'est futile! Alors que c'est primordial! Seulement, il faut faire attention. Lorsqu'on a la prétention, comme moi, d'entraîner les gens dans l'imaginaire, il faut pouvoir les ramener dans le réel ensuite... et sans dommage. C'est une responsabilité ${ }^{4}$. 


\section{Géopolitique du Moyen-Orient : le tourisme face aux diversités et aux adversités}

Le Moyen-Orient est une région célèbre doublée d'une réalité méconnue. La raison? C'est apparemment l'absence de celle-ci qui fait défaut à toute compréhension fondée sur le discernement objectif de la situation régionale contemporaine ! || paraît que l'attrait culturel et confessionnel qu'exerce cette région sur les gens du Livre (cf. les trois religions célestes) opère une dérive sélective, faisant qu'une majorité d'observateurs ne s'intéresse, au grand dam de la neutralité équitable, qu'à ce qui confirme ses certitudes, de peur de perdre ses repères, ses dogmes, voire ses croyances.

Vu de près, ou plutôt d'une contrée limitrophe, basée à l'intersection de l'Orient et de l'Occident ${ }^{5}$, le Moyen-Orient constitue une zone d'ombres et de lumières, suffisamment éclairée par endroits, mais mal éclairée dans l'ensemble, malgré une activité médiatique internationale intense. Dans ce fameux carrefour sociogéographique de toutes les convoitises et de tous les métissages (géopolitique, socioéconomique, ethnolinguistique...), les aveuglements sont fréquents. Le malaise est profond. D'une part, les nations faibles sur le plan militaire sont hantées par le sentiment chronique d'être des « victimes » agressées sans cesse; d'autre part, les grandes puissances ayant des intérêts dans la région sont habitées par une obsession de "démocratie » civilisatrice, prête à l'exportation. Peut-être n'est-il pas inutile de se demander pourquoi? "L'existence avérée des plus grandes réserves pétrolières de la planète dans cette zone confère aujourd'hui au Moyen-Orient une place straté- gique de premier plan dans le dispositif géopolitique de l'Occident » (Rigoulet, 1993 : 1058-1068). Dans ce contexte, le tourisme peut attendre. C'est probablement la seule activité viable et rentable de la période postpétrole.

\section{Cadre géographique et sociopolitique du Moyen-Orient : ou comment prendre " ces déserts " pour des réalités !}

Le Moyen-Orient appartient à la vaste zone climatique aride qui s'étend de part et d'autre du tropique du Cancer $\left(23^{\circ} 27^{\prime} \mathrm{N}\right)$.

Une grande partie du territoire consiste en déserts et en steppes dont il existe, à vrai dire, toutes les variétés, de la maigre steppe semi-désertique d'Arabie aux étendues herbeuses du nord du Kazakhstan, aptes à nourrir d'immenses troupeaux. Dans un tel contexte, les massifs montagneux jouent un rôle déterminant car ils provoquent des précipitations et approvisionnent régulièrement en eau leurs environs (Sellier et Sellier, 2004: 11).

Sur le plan biogéographique, il dispose d'un climat très favorable, de paysages remarquables et d'un patrimoine historique d'une richesse inégalable.

La délimitation du Moyen-Orient, sur le plan sociogéographique, ne fait pas l'unanimité, loin s'en faut. Même les ouvrages qui font autorité dans le monde des écrits de géopolitique contemporaine ne s'accordent ni sur les limites de la région ni sur le nombre de pays qui

Tableau 3

Le Moyen-Orient, vu de l'extérieur et vu de l'intérieur

Vu de l'extérieur

Vu de l'intérieur

«Vu de l'extérieur, le Moyen-Orient est une charnière entre la Méditerranée et l'océan Indien, entre l'Europe et les grands pays d'Asie (Inde et Chine...), entre la Russie et les mers chaudes. [...] Aujourd'hui, on considère comme un tout l'espace situé à l'est de la Méditerranée. » C'est aussi le foyer de la foi et de la culture judéo-chrétienne, un immense réservoir pétrolier du monde et une destination touristique de rêve. la composent. "Aujourd'hui on considère comme un tout l'espace situé à l'est de la Méditerranée. Aussi le 'Moyen-Orient' - non sans excès de langage - inclut-il le 'ProcheOrient' » (Sellier et Sellier, 2004 : 11).

L'expression « Moyen-Orient » a été inventée par les Britanniques au début du XXe pour désigner une zone-tampon entre l'empire ottoman et l'empire des Indes alors sous leur domination. Constitué de royaumes, de républiques, d'émirats et de sultanats créés en majorité de toutes pièces au début du même siècle sur les ruines de l'empire ottoman, sans grande homogénéité sociogéographique ni cohérence sociopolitique, le Moyen-Orient constitue un bloc hétéroclite. Ce puzzle préfabriqué est composé en grande majorité d'États musulmans appartenant à des groupes ethnolinguistiques distincts.

Le Moyen-Orient se définit ainsi en termes non plus géostratégiques mais religieux et ethniques. En font partie les peuples iraniens (dont l'aire déborde sur le Pakistan) et la plupart des peuples turcs, ce qui incite à rattacher l'Asie centrale au Moyen-Orient, comme le veut toute l'histoire antérieure au XIXe siècle et comme le permet aujourd'hui la disparition de l'URSS. En font partie aussi les peuples arabes, sauf ceux du Maghreb, à l'évidence situés hors de l'espace moyen-oriental. Enfin, pour des raisons géographiques et historiques, se trouvent inclus dans l'ensemble des populations non musulmanes: les Géorgiens, les Arméniens, les minorités arabes demeurées chrétiennes, les Juifs d'Israël (Sellier et Sellier, 2004 : 11).

En fait, on n'a jamais assez insisté sur le fait que seul le dialogue, interculturel et interconfessionnel, permet de caresser l'espoir de voir, à moyen terme, la paix reprendre ses droits et régner au Moyen-Orient, au profit d'un brassage enrichissant des cultures et des populations dans un élan de rapprochement apaisant entre les peuples. Vu sous cet angle, le tourisme a un rôle hautement pacifique, certains diront humanitaire, à jouer dans cette région de tous les risques et de tous les espoirs aussi. N'en déplaise aux pessimistes, cet espace de divergences politiques, de conflits économiques et de querelles théologiques est aussi un pôle d'émergences démocratiques et d'affluences touristiques.

Source : D'après Jean et André Sellier (2004 : 11). 


\section{Patrimoine socioculturel et historique du Moyen-Orient : un gisement touristique en temps de paix}

Les recherche archéologiques ont révélé que:

I'homme est établi au Proche-Orient depuis au moins un million et demi d'années, date estimée des traces les plus anciennes retrouvées à Ubeidiyeh au sud du lac Tibériade. C'est l'espèce dite Homo Erectus qui s'aventure la première hors du continent africain et, franchissant la péninsule du Sinaï, vient peupler l'Asie et l'Europe (Caubet et Poueyssegur 2001 : 20).

De là à dire que cette vague de migration "originelle", la plus ancienne connue de I'humanité, constitue un embryon touristique primitif, il n'y a qu'une hésitation, mais il y faudrait des preuves tangibles ou, à défaut, des convictions dialectiques. N'empêche que cette première grande migration de l'histoire de l'humanité s'inscrit dans le cadre d'un voyage intercontinental! Alors, pourrait-on savoir un jour pour quelle raison ces premiers "globe-trotters", vêtus de peaux et armés de pierres taillées, ont décidé de quitter leur terre natale? Sont-ils partis par plaisir ou par contrainte? Ou bien va-t-on continuer à penser que le continent africain a toujours été, et continue à être fatalement, une terre répulsive?

Toutefois, il a fallu attendre très longtemps avant de voir ces "voyageurs primitifs " se muer doucement en scientifiques habiles. Ce n'est que vers 15000 av. J.-C., c'està-dire à la fin des dernières glaciations, que les premiers signes d'une civilisation intelligible ont commencé à essaimer. L'Homme quitte les grottes, les falaises et les abris naturels et s'installe dans le "croissant fertile» au cœur de la Mésopotamie. Vers 12500 av. J.-C., " des groupes entiers commencent à s'établir collectivement dans de petites unités d'habitat sédentaire : les premiers villages " (Caubet et Poueyssegur 2001 : 20). Vingt-cinq siècles après l'apparition des premiers citadins de notre planète, I'habitat maçonné (-10 000 ans) fait son apparition alors que « l'agriculture est apparue au ProcheOrient, pour la première fois dans I'histoire de l'humanité, entre 8000 et 6000 av. J.-C. » (Sellier et Sellier, 2004 : 12).
L'invention de l'écriture en Mésopotamie va faciliter les échanges commerciaux et permettre le passage de l'apprentissage par mimétisme (tradition) à l'apprentissage cognitif (écriture et lecture), grâce à la transmission du savoir par quelques bribes d'instruction. «De Sumer et d'Akkad vient l'écriture qui, au IVe millénaire avant notre ère, sur le sol de l'Irak actuel, donne naissance à la raison déductive et rend possible une religion universelle » (Bottéro et al., 1998: $4^{e}$ couverture). Dès lors, «l'économie de prédation fait place à une économie de production : l'homme prend possession du milieu naturel » (Caubet et Poueyssegur 2001 : 23). On peut penser que le passage de la chasse et de la cueillette à la vie sédentaire a déjà produit sous une forme ou sous une autre un type de voyage raisonné qu'il faut, évidemment, replacer dans le contexte de l'époque. «Les plus anciennes représentations de la roue datent environ du IV ${ }^{\mathrm{E}}$ siècle avant Jésus-Christ. On les a retrouvées en Asie mineure. II semble que le char à roues ait été utilisé dès l'an 3000 avant Jésus-Christ en Mésopotamie» (Sigaux, 1965 : 8). L'accélération des découvertes, par la suite, a sûrement poussé des femmes et des hommes à voyager pour écouler ou pour acquérir (en recourant au troc) les produits des nouvelles créations ou inventions. Ce n'est pas encore le tourisme d'affaires, mais le voyage a déjà quelques relents commerciaux.

Du nomadisme au tourisme, l'Homme ne fait que perpétuer l'amour du mouvement. De ce fait, la stabilité, mère de la civilisation selon lbn Khaldoun ${ }^{6}$, peut paraître une "anomalie " porteuse de progrès. Celui-ci semble n'apporter que le confort matériel individuel, mais pas le reste, c'est-à-dire la quiétude socio-spirituelle nécessaire à l'apaisement de l'âme. De ce bien-être offert par le progrès, la liberté demeure l'acquis fondamental des pays développés. C'est justement cette jonction entre liberté, confort et contrainte qui fait partir momentanément et qui permet à l'imaginaire de susciter le voyage et à ce dernier d'alimenter convenablement le tourisme.

Se délier pour mieux goûter la proximité des choses. Sans forcément en avoir conscience, tout un chacun fait cela dans la vie quotidienne: voyages, tourisme, retraites, cures, "breaks» de tous ordres, nombreuses sont les occasions de divers genres où l'on largue les "amarres », où l'on s'exile et fuit afin de redonner de la saveur à ce qui, sous les coups de boutoirs de la routine, n'en a plus guère (Maffesoli, 2003 : 37).
Toujours est-il cependant que certains touristes considèrent leur visite dans la région située entre la Méditerranée et la Mésopotamie comme un retour sur des lieux perçus comme des foyers de l'éclosion de l'intelligence humaine. Ce type de voyage procède de l'inversion des rôles, puisque ce qui est sensé être un "aller» est en fait, selon le touriste fervent, un « retour ». Comme quoi, le voyage par nostalgie millénaire des temps et des lieux fondateurs de la civilisation humaine relève d'une problématique existentielle doublée d'une motivation exceptionnelle.

\section{Fondements et développement de l'imaginaire touristique du Moyen-Orient : quand le présent dépend du passé}

Le Moyen-Orient évoque, confusément pour les uns et clairement pour d'autres, les civilisations antiques et les voyages romantiques. D'autres images se superposent et brouillent la vision des analyses simplistes. Ces images d'ordre historique (patrimoine socioculturel), économique (deux tiers des réserves mondiales de pétrole) et géopolitique (luttes d'influences) cohabitent difficilement dans les esprits et dans l'espace. Elles contribuent à tisser des imaginaires plurivalents. Ceux-ci s'inspirent des fondements culturels des peuples sur lesquels le socle religieux a une emprise latente, mais se nourrit continuellement des événements contemporains et des interprétations qu'en font les auteurs et les commentateurs de l'information.

Dans le cas du Moyen-Orient, les avis prolifèrent. Ce qui est une bonne chose. Ce qui n'est pas bien, par contre, c'est l'absence de tribunes ${ }^{7}$ libres, objectives et neutres pour assainir ces avis et décloisonner ${ }^{8}$ les savoirs qui traitent de cette région sensible au sein de laquelle l'opinion serait aujourd'hui collective, populiste et normative. Or, par le passé, elle était, habituellement et sociologiquement, stratifiée en trois niveaux : local (incorporant résidentiel ou tribal), régional (identité communautaire ou ethnique) et national (appartenance patriotique ou nationaliste). Alors peut-on, pour ce qui est du Moyen-Orient, dissocier l'imaginaire touristique des considérations et des préoccupations géopolitiques et socioéconomiques? 
D'une manière très simplifiée, trois types d'imaginaires touristiques collectifs semblent dominer :

- Les peuples indochinois et russo-mongols, malgré le facteur du voisinage, ont une image quelque peu profane du Moyen-Orient. La spiritualité « non céleste » des croyances et des confessions (à l'exclusion des communautés musulmanes et judéo-chrétiennes) de ces peuples d'Extrême-Orient a développé un imaginaire souple fortement imprégné de perceptions bouddhistes, sans liens avec les révélations célestes, donc propre à la méditation transcendantale. Cela explique leur détachement relatif vis-à-vis des approches divines du Proche et du Moyen-Orient.

- Les pays occidentaux ont apparemment un imaginaire tissé de nostalgie dont se nourrissent les valeurs judéo-chrétiennes d'origine proche-orientale. Cependant, la qualité et la généralisation de l'enseignement, plus ou moins scientifique, atténuent, en les modulant, les fondements et les référentiels de ces valeurs. Sans être débarrassés complètement de croyances légendaires (heureusement pour le tourisme), ces enseignements permettent de laisser une place conséquente aux représentations moins fétichistes et moins défaitistes, mais non dépourvues, malheureusement, de sentiments de supériorité.

- Les peuples du Maghreb et du MoyenOrient ont sûrement un imaginaire composite difficile à saisir pour diverses raisons : d'abord parce qu'ils sont acteurs, malgré eux, des événements imposés à la région, tout en demeurant spectateurs lorsqu'il s'agit de prendre des décisions déterminantes pour son avenir. De plus, partagés entre des appartenances mâtinées d'ordres ethnique, confessionnel et idéologique, sans liberté d'expression ni d'action dans la plupart des cas, ces peuples ont des préoccupations autrement défensives et n'accordent au tourisme, en tant que fils de la prospérité et de la liberté, qu'une attention circonstancielle. II ne faut pas oublier que l'imaginaire touristique germe et fleurit là où la paix et la prospérité cultivent l'épanouissement par le développement.

Il est vrai que le tourisme a bouleversé énormément de mœurs et d'habitudes en amont (pays émetteurs) et en aval (pays récep-

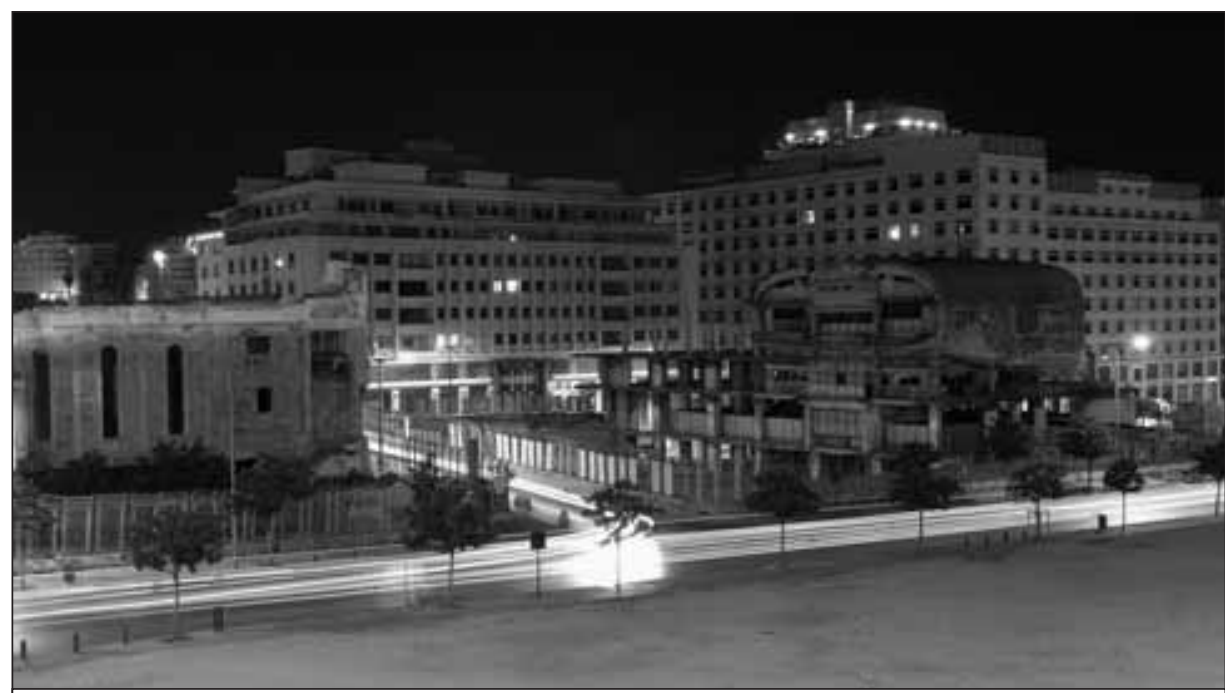

La reconstruction de Beyrouth après la guerre civile.

Photo: Saër Karam

teurs) des aires de son déploiement. Cela en est même révolutionnaire lorsqu'il arrive à bousculer l'ordre établi. « La vacance des valeurs produit la valeur des vacances » (Edgard Morin cité par Dumazedier, 1990 : 189-198). Voilà qui remet les pendules à l'heure pour ce qui est des politiques normatives où la pudeur imposée et le pouvoir autoritaire règnent en maîtres absolus sur les esprits. « La libération nationale a eu pour effet pervers de bannir de l'univers mental des élites gouvernementales l'idée de l'État de droit. » (Kassir, 2004)

Le tourisme est, dit-on, responsable de la grande mutation à soi-même. Mais quelle est la part de l'imaginaire dans ces bouleversements sociétaux profonds attribués au tourisme? Au regard de cette mutation, " une part de l'individualité qui était cachée, contenue ou réprimée, s'exprime plus librement. Ce n'est pas la fin du contrôle social, des déterminants sociaux en tous genres, des règles de bienséance de l'institution, mais les frontières de celui-ci ont reculé " (Dumazedier, 1990 : 189-198). Ce constat, valable dans les pays occidentaux, n'en est qu'à ses débuts dans quelques pays du Maghreb et du Moyen-Orient. C'est que la pesanteur classique cantonne encore l'individu dans une sphère sociale imperméable faite d'une triple cloison : sociale, politique et religieuse. Dans ce cas, l'imaginaire n'est pas toujours le fruit d'un débordement émancipateur ou créateur et ne résulte, fréquemment, que d'une propagation directive. En fermant ses frontières devant la jeunesse de ces pays, l'Occident ne fait qu'aggraver une situation déjà pénible.
Ayant incontestablement des composantes essentiellement culturelles, l'imaginaire des peuples du Tiers-Monde est d'essence collective. Car il n'est pas toujours facile de s'extraire de sa communauté d'appartenance ou d'affiliation pour pouvoir porter un regard neutre et serein sur autrui et sur tout et, par une sorte de ricochet introspectif, sur soi-même. Tout regard porté sur l'Autre est conditionné par le modèle socioculturel pré-acquis basé a priori sur la solidarité organique. La récusation ou la critique s'oriente facilement vers des centres d'intérêt exogènes. II faut sûrement être imprégné d'une culture générale à la fois importante et polyvalente, ouverte et tolérante (autrement dit, il faut voyager, ce qui n'est pas le cas), pour être en mesure d'échapper à la pesanteur sociale, culturelle, idéologique ou théologique de son milieu d'origine et disposer, en conséquence, d'un imaginaire propre.

Aujourd'hui, l'opinion locale et régionale est presque corporatiste, voire unilatérale. La chute du communisme, l'échec du nationalisme et la déception du libéralisme, tous trois d'obédience exogène ou d'importation, poussent les peuples, laminés par les guerres coloniales ainsi que par les retards socioéconomiques et excédés par la succession des défaites face aux hégémonies extérieures (défaites aggravées par les oppressions de leurs régimes inamovibles), à un retour populaire et populiste aux sources : la foi. La religion offre une alternative prometteuse et constitue, à la fois, une tribune d'expression, un catalyseur de masses et un grand espoir d'avenir et, au-delà, au sens propre et au sens figuré, un espoir pour l'au- 
delà. Ce corporatisme, nourri par un endoctrinement permanent souvent mal assimilé parce qu'il se veut vengeur, intronise la méfiance en tant que dispositif naturel permanent. Et c'est régulièrement le tourisme, la face locale d'un Occident lointain et donc inabordable, qui en fait les frais. Dans ce contexte de psychose pessimiste, l'imaginaire subit le contrecoup du repli des sociétés du Moyen-Orient sur elles-mêmes et se débarrasse de ses apparences ludiques pour ne garder que les désirs fantasmatiques aux relents paradisiaques, saupoudrés de quelques plaisirs chastes.

\section{Mille et un souvenirs de lieux rêvés pour des visites différées : une certaine idée du Moyen-Orient}

Les croisés ${ }^{9}$ sont probablement les premiers "globe-fighters" à ramener en Europe des souvenirs et des images du Proche et du Moyen-Orient. Des souvenirs peut-être douloureux, mais des chroniques qui ont, tout même, contribué à faire connaître l'importance, non seulement théologique, mais aussi historique, de cette terre dédiée par la synergie divine et humaine à la révélation des religions célestes. Les peuples du Proche et du Moyen-Orient ont, eux aussi, gardé des traces et des images de cette période du Moyen-Âge. II faut rappeler, en insistant sur la nature de l'époque, que "la route n'est qu'une trace établie par l'usage» (Boyer, 1996 : 23). C'est dire que «les périls les plus lointains, souvent aussi les plus dangereux, sont ceux qui semblent avoir attiré le plus grand nombre de curieux [...] » (Sigaux, 1965: 43). II suffisait à ceux qui s'aventuraient au Proche et au Moyen-Orient à l'époque, avec ou sans route tracée, de se fier à sa perspicacité de voyageur contrit pour s'orienter et à son instinct de vagabond érudit pour se défendre.

Le voyage, ainsi que les imaginaires, les rêves et les autres fantasmes sociaux, est un filon caché parcourant l'ensemble du corps social. Il ressemble à ces cristallisations lumineuses enfouies au cœur de la roche, et que le chercheur d'or ou de pierres précieuses trouvera, au bout d'un long labeur, après avoir remué des tonnes de minerai sans aucune valeur » (Maffesoli, 2003: 15).
Dans les pays arabo-musulmans de la région, où les défaites se succèdent et se ressemblent, des récits teintés de gloire ont longtemps meublé la littérature orale populaire. Inutile de rappeler que ces contes et chroniques populaires hautement prisés par les jeunes en mal de victoires et de triomphes valorisants constituent le noyau dur des fondements structurants de l'imaginaire collectif.

En Europe occidentale, après la Renaissance, l'émergence de l'orientalisme, malgré des pillages excessifs, a contribué à l'amélioration de l'image du Moyen-Orient par une sorte de sacralisation de son patrimoine au point de provoquer (fin XVIII - début $X I X{ }^{e}$ siècle), une sorte de «moyen-orientomanie». Ce mouvement hautement intellectualisé est matérialisé par des productions historiques, géographiques et artistiques qui émanent de grands explorateurs ou voyageurs. Ces moments sont restitués, image à l'appui, par les grands maîtres de la peinture; et l'on comprend que cette région "phare » offre, de nos jours, aux Hommes de notre époque quelque peu « déshumanisée » par l'importance des relations et des considérations matérielles, une pléthore d'images et d'imaginaires qui donnent à tout un chacun l'opportunité de s'y retrouver: au croyant la possibilité de purifier son âme, à l'intellectuel l'éventualité de visualiser ses théories et au profane une bonne occasion de s'élever par la visite in situ au rang de l'émerveillement. «En fait, tout cela est une manière de fuir la grégaire solitude propre à l'organisation rationnelle et mécanique de la vie sociale moderne » (Maffesoli, 2003 : 33).

Comme toute utopie naissante mais dominante, l'imaginaire propagé par une représentation surfaite du Moyen-Orient est meublé au fil des années par l'apport d'ingrédients allégoriques liés au courant des artistes et des hommes de lettres admirateurs et promoteurs du Levant. "Peu à peu, le Moyen-Orient et l'Afrique trouvent leur place aux côtés de la Grèce, de l'Italie et de l'Égypte. L'Itinéraire de Paris à Jérusalem de Chateaubriand, publié en 1811, le Voyage en Orient de Lamartine (1835), puis celui de Nerval (1851), trouvent un écho dans la peinture de Delacroix et des orientalistes » (Patin, 2005 : 18). La grâce des scènes où la femme en chair et en couleur, entre esclave, maîtresse et princesse, a semé longtemps la confusion dans les esprits, souvent préparés par les contes des Mille et Une Nuits à bondir dans le sens d'un exotisme fantasmé, sans prendre, la plupart du temps, les précautions d'usage. II est inévitable que les scènes de harem et les images de danse du ventre se soient glissées en travers de l'opinion des masses, des opinions mal assainies par un contexte éducatif qui peine à se débarrasser des reliquats abstrus du MoyenÂge ; c'est ainsi que des rêveurs utopiques ont fini, sous l'effet d'une fantaisie sociale (toutes proportions gardées), par prendre leurs désirs pour des réalités et par se lancer dans la conquête, non pas du rêve, mais de l'illusion. Mais, derrière ces rêveurs et des penseurs, se profilent des desseins coloniaux où le rêve est évacué violemment par le calcul.

À partir de ce moment, la nature des expéditions prend une autre allure : intellectuels, religieux et scientifiques (souvent au service ou complices des militaires) vont sillonner le Moyen-Orient à la recherche d'antiquités rares ou exceptionnelles.

Les vues de Palmyre (Syrie) ou de Baalbek (Liban) qui décorent Le voyage en Syrie et en Égypte, publié en 1787 par le comte de Volney (Constantin François de Chassebœuf), reproduisent très fidèlement les illustrations des ouvrages consacrés à ces sites trente ans plus tôt par les Anglais Robert Wood et James Dakwins (Patin, 2005: 14).

À ce propos, il faut préciser que ces illustrations ont colporté suffisamment de réalisme, et d'illusions aussi, pour que des générations entières de penseurs et d'intellectuels aient accompli, sous forme d'obligation morale, le voyage du MoyenOrient, en quête de ressourcement intellectuel ou spirituel. Certains ont même espéré y trouver l'inspiration féconde et indispensable à la conquête d'une célébrité qui tardait à venir, eu égard aux ambitions d'une aristocratie malmenée par les débuts de la révolution industrielle.

Dès le début du XIXe siècle, "la concurrence est vive entre Français, Allemands (Prussiens) et Britanniques qui rivalisent pour acquérir des antiquités prestigieuses. [...] Dans les pays qu'il conquiert, Bonaparte prélève des ensembles considérables d'objets d'art » (Patin, 2005 : 15). L'exposition de ces objets artistiques dans les musées européens a contribué à l'émergence d'un intérêt particulier pour le Moyen-Orient, sans pour autant démystifier 
une image de fond qui fait de la région une contrée attirante par ses richesses et attrayante par son exotisme, mais rebutante par son fatalisme!

Par la suite, le progrès soutenu par la révolution des technologies de l'impression et la facilité des voyages (cf. moteur à vapeur) va permettre la généralisation et la vulgarisation des outils et des moyens d'information. "Mis au point par l'Allemand Senefelder à l'extrême fin du XVIII ${ }^{e}$ siècle, le procédé d'impression de la lithographie contribue à développer considérablement le genre du 'voyage pittoresque', véritable inventaire du patrimoine naturel, archéologique et architectural » (Peltre, 2004 : 79). L'apparition de la photographie en 1839, procédé mis au point par les frères Lumière à Lyon, va révolutionner, un peu plus tard, le voyage d'exploration anthropologique et géographique. La peinture et la photographie ont joué un rôle décisif dans la connaissance touristique du Moyen-Orient.

Si «le voyage pittoresque » devient avec le temps moins recherché, l'illustration garde son actualité dans les revues consacrées à l'exploration géographique, comme Le Magasin pittoresque ou Le Tour du monde, nouveau journal des voyages, créé en 1860, auxquels collaborent de nombreux orientalistes (Peltre, 2004 : 83).

En fait, le tourisme au Moyen-Orient ne peut pas être dissocié des autres activités et vocations régionales. Et si, aujourd'hui, il figure au rang des secteurs négligés, il risquera à l'avenir de prendre la tête des secteurs clés dans l'économie moyenorientale. Cette dernière est biaisée par la défense ou la recherche des intérêts particuliers, que ce soit de la part des puissances extérieures ou de celle des régimes locaux. Ces économies rentières ne permettent aujourd'hui qu'un développement touristique subsidiaire ou à peine convenable (cas des pays méditerranéens de la région) et rares sont ceux qui s'apprêtent à entrer de plain-pied dans l'ère du "capitalisme post-rentier ». Les relations entre l'État et la société au Moyen-Orient souffrent d'une dysmorphie atavique d'ordres géographique (frontières artificielles), sociopolitique (États préconçus) et économique (rentes pétrolières).
Les pays producteurs de pétrole, quant à eux, n'ont développé qu'un tourisme d'affaires ponctuel. Leurs recettes en pétrodollars ont, bien entendu, permis une sélection en faveur d'une hôtellerie " haut de gamme». Les soucis et autres tracasseries de la rentabilité ne se sont nullement posés, du moment que le budget de l'État était disposé à accorder les rallonges nécessaires pour conforter l'embellissement de l'image extérieure du pays. Cette image et tant d'autres, aussi artificielles les unes que les autres, ont longtemps voilé le fonctionnement de systèmes politiques d'un autre âge (Hillali, 2003 : 35).

\section{Conclusion}

Le Moyen-Orient et sa part de rêve cheminent dans l'imaginaire des candidats au grand voyage par une sorte d'alchimie transcendante. La renommée historique de cette région aux facettes multiples a la particularité d'en faire un pôle d'invitation permanente au voyage. En conséquence, l'esprit se porte en éclaireur des lieux moyen-orientaux et s'offre le luxe d'aller faire une reconnaissance des endroits de mémoire et, fait peu déontologique, il arrive qu'il se brouille sous l'effet de charges émotionnelles et impose aux passionnés sa propre vision de l'histoire et de la géographie régionale et parfois sa " conception » du voyage ! Et l'on comprend que, bien que partiellement, la réalité moyenorientale fasse, malheureusement, que la passion conteste constamment à la raison toute légitimité d'interférence. Dans ce cas précis, l'imaginaire dérive et le tourisme devient alors la première victime de ce qui est censé à être le rapprochement entre les peuples et le dialogue entre les cultures.

Le problème de l'imaginaire dans sa relation au voyage moyen-oriental, c'est qu'il est tenace ou diffus. II se situe sur la marge floue du rêve, d'un espace imaginé (le pays récepteur), tout en ayant son ancrage dans le territoire de départ (pays émetteur)! Donc, entre l'imaginaire du candidat au voyage, l'espoir du partant et la réalité du visiteur sur place, il y a toujours un espace-temps flou, qu'il va falloir clarifier sans altération pour rapprocher la vision du « visiteur » de celle du « visité ». Mais qui se soucie en réalité de ces détails, situés le plus souvent aux antipodes les uns des autres?
On se trompe ou l'on pense contourner le problème quand on cherche à faire croire que le malaise est purement socioéconomique. Apparemment, la théologie et l'idéologie, dans leurs manifestations extrêmes, ont quelque part des intérêts communs, même si cela paraît à première vue paradoxal. Elles prônent la défense de la morale et l'obéissance à des règles propres à chacune (et c'est le point de discorde), quitte à piétiner la réalité... une réalité peuplée d'hommes, de femmes et d'enfants. Cela explique que les antagonismes ont, par moments et par endroits, des raisons que la raison ellemême ignore! Les appétences sont alors attisées par l'absence de réalisme autochtone, qui laisse grandes ouvertes les portes à l'hégémonisme de tous les opportunistes. Et à force de brandir des vérités ou des convictions arrêtées, les extrémismes ne donnent au dialogue qu'une chance très minime et écartent inopportunément l'émergence de la sérénité attendue de toutes les parties en présence. "Le sacré peut être à l'origine d'expériences sublimes entrainant des gestes héroïques et des sommets d'humanité et de sagesse. II peut aussi devenir une source de fanatisme, d'intolérance et d'étroitesse d'esprit menant à des erreurs et à des aberrations funestes » (Tessier et Prades, 1991: 12). Dans ce cas, l'imaginaire constructeur cède la place au fantasme destructeur.

Aussi, est-il regrettable de constater que le touriste "raisonné ", le visiteur passionné et l'homme d'affaires se croisent régulièrement dans les avions et les hôtels, mais rarement sur les hauts lieux de l'histoire. Et c'est bien dommage! Alors, laissons les affaires aux affairistes et saluons la volonté des touristes qui choisissent d'aller marcher sur les traces de ceux qui ont initié la civilisation humaine.

Mimoun Hillali est professeur à l'Institut supérieur international du Tourisme (Tanger, Maroc), est l'auteur du Tourisme international vu du Sud, publié aux Presses de l'Université du Québec.

\section{Notes}

1 Proverbe arabe "Ma a'diaqa al aïcha laoula fous'hata al amal ", cité par Hillali (2003). 
2 Au sens de la "production de l'imagination par laquelle le moi cherche à échapper à l'emprise de la réalité ", d'après le Petit Robert (dictionnaire), 1996, p. 892.

3 Le tourisme d'affaires échappe lui aussi à la notion d'agrément dans sa dimension récréative et presque à but non lucratif.

4 D'après un sketch de Raymond Davos, comédien français.

5 Le Maroc est considéré comme le plus occidental des pays de l'Orient musulman.

6 Homme d'État et juge musulman (Cadi), d'origine andalouse, né à Tunis en 1332 et mort au Caire en 1406.

7 Les mass média peinent à soutenir l'objectivité à force d'être soutenus, euxmêmes, par des créanciers ou des courants idéologiques et ils perdent toute crédibilité à force d'orienter l'information.

8 Je tiens personnellement à saluer l'initiative de Téoros d'avoir choisi cette région comme thème du présent volume.

9 Le mot "croisé" traduit l'engagement de ceux qui, à l'appel du pape [Urbain II, en novembre 1095, au terme d'un concile tenu à Clermont-Ferrand], réagirent en criant "Dieu le veut! " et en faisant coudre aussitôt sur leur vêtements des croix, exprimant par là qu'ils faisaient vœu de répondre à son appel (Flori, 2001: 229-230).

\section{Bibliographie}

Bottéro, Jean, Clarisse Herrenschmidt, et JeanPierre Vernant (1998), L'Orient ancien et nous, Paris, Éditions Hachette.

Boyer, Marc (1996), L'invention du Tourisme, Paris, Éditions Gallimard, coll. « Art de vivre ».

Caubet, Annie, et Patrick Poueyssegur (2001), L'Orient ancien (aux origines de la civilisation), Paris, Édition Pierre Terrail.

Dortier, Jean-François, et Testot Laurent (2005), La religion, unité et diversité, Auxerre, Éditions Sciences humaines.

Duby, Georges (1996), Atlas historique, Paris, Éditions Larousse-Bordas.

Dumazedier, Joffre (1990), "Vacances et valeurs ", dans Les vacances, un rêve, un produit, un miroir, Autrement, Série « Mutations ", no 111, janvier, p. 189-198.

Flori, Jean (2001), Guerre Sainte, jihad, croisade, Paris, Éditions du Seuil.

Hillali, Mimoun (2003), Le tourisme international vu du Sud, Essai sur la problématique du tourisme dans les pays en développement, SainteFoy, Presses de l'Université du Québec.

Kassir, Samir (2004), Considérations sur le malheur arabe, Éditions Actes Sud, coll. « Sindibad ».

Lacoste Yves (dir.) (1993), Dictionnaire géopolitique, Paris, Édition Flammarion, p. 1058-1068.

Lauru Gabs, Didier, Janine Gabillet, et Patricia Berriau (2004), Tout est psy en vacances, Paris, Éditions Albin Michel.

Louis, René (1990), Les Vacances: Un rêve, un produit, un miroir, Autrement, série "Mutations ", $n^{\circ} 111$, janvier, p. 11.
Maffesoli, Michel (2003), Le voyage (ou la conquête des mondes), Paris, Éditions Dervy.

Miossec, Jean-Marie (1995), "Tourisme et loisirs au Maghreb et au Moyen-Orient: L'explosion d'un besoin ", dans Jean-François Troin (dir.), Maghreb - Moyen-Orient - Mutations, coll. "Dossiers des images du monde ", SEDES, p. 251-290.

Organisation mondiale du tourisme (1999), Middle Est, Tourisme Market Trend, Madrid.

Organisation mondiale du tourisme (2002), Annuaire des statistiques du tourisme, Madrid, vol. 1 et 2.

Organisation mondiale du tourisme (2005), Baromètre du tourisme mondial, Madrid, vol. 3, n 3 , octobre.

Patin, Valéry (2005), Tourisme et patrimoine, Paris, La Documentation française.

Peltre, Christine (2004), Orientalisme, Paris, Éditions Pierre Terrail.

Rigoulet, David (1993), Concessions et frontières au Moyen-Orient, dans Yves Lacoste (dir.), Dictionnaire de géopolitique, Paris, Édition Flammarion.

Sellier, André, et Jean Sellier (2004), Atlas des peuples d'Orient (Moyen-Orient, Caucase, Asie centrale), Paris, Éditions La Découverte.

Sigaux, Gillbert (1965), Histoire du Tourisme, Genève, Éditions Rencontre.

Tessier, Robert, et José A. Prades (1991), Le sacré. Éditions du Cerf / Éditions Fides.

Theum, Nadia (2005), Le tourisme en Méditerranée, une perspective socioculture/le, Aix-en-Provence, Edisud.

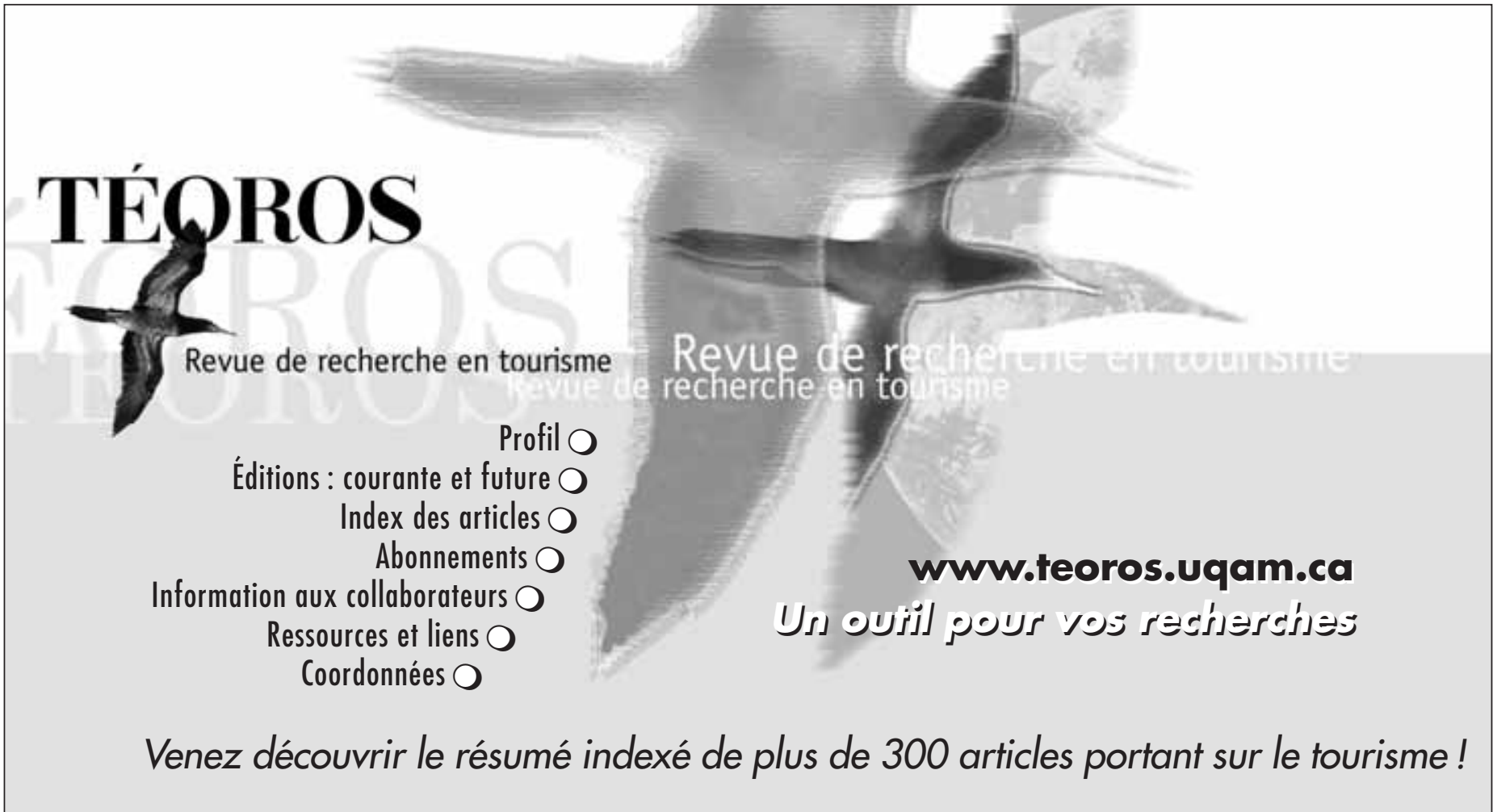

